\title{
Absorbing boundaries in the mean-field approximation
}

\author{
Chirag Jhala, Ingo Dreissigacker, and Manfred Lein \\ Institut für Theoretische Physik and Centre for Quantum Engineering and Space-Time Research (QUEST), Leibniz, Universität Hannover, \\ Appelstraße 2, D-30167 Hannover, Germany \\ (Received 16 September 2010; published 27 December 2010)
}

\begin{abstract}
Absorbing boundaries in the mean-field approximation are investigated and applied to small systems interacting with strong laser fields. Two types of calculations are considered: (i) a variational approach with a complex absorbing potential included in the full Hamiltonian and (ii) the inclusion of a complex absorbing potential in the single-particle equations. It is elucidated that the second approach outperforms the variational approach for small grids.
\end{abstract}

DOI: 10.1103/PhysRevA.82.063415

PACS number(s): $33.80 . \mathrm{Rv}, 31.15 . \mathrm{xt}, 31.15 . \mathrm{ee}$

\section{INTRODUCTION}

The time-dependent Schrödinger equation, although describing all the relevant nonrelativistic physics of atoms and molecules in strong laser fields [1], is too complex and time consuming to solve for all but very small systems. The difficulty arises from the exponential increase of computational times with an increasing number of particles. Furthermore, the large excursions of laser-driven electrons require large numerical grids or basis sets. The time-dependent Hartree (TDH) method [2], which approximates the wave function as a product of single-particle orbitals seems to be a practicable approach. Via a variational principle, the TDH approximation results in a set of coupled single-particle equations of motion which are quite simple to solve. Each particle moves in its effective potential, which includes the mean field generated by all other particles. An alternative effective-potential method is provided by time-dependent density functional theory (TDDFT) [3]. TDDFT is applicable to systems of identical or nonidentical particles. The formal framework for the latter case is known as multicomponent density functional theory $[4,5]$. To reproduce the density of an interacting many-particle system, Kohn and Sham (KS) introduced an auxiliary system of noninteracting particles [6], originally to treat ground-state problems. The dynamics of the KS system is governed by the KS potential, which consists of the Hartree, exchange, and correlation potentials. If one neglects the exchange-correlation potential, one arrives at the simple Hartree TDDFT method. We note that this method when applied to a system of identical particles includes unphysical self-interactions since the TDDFT Hartree potential is the mean field generated by all particles. In this respect, the method differs from the Hartree method derived from the variational principle. Below, however, we consider the following special cases: (i) a system with two distinguishable degrees of freedom and (ii) a two-electron system. In both cases, it is natural and straightforward to omit any self-interaction terms in the equations of motion.

After choosing one of the methods, one faces the problem that solving the equations of motion may still require very large numerical grids. While, for example, the description of high-order harmonic generation [7] generally works well with moderately sized grids, the situation is different for

*Chirag.Jhala@itp.uni-hannover.de above-threshold ionization spectra [8], which require the knowledge of the entire final-state wave function. Therefore, one must choose the grid size depending on the nature of calculation to be performed. Numerical simulations on a small grid are hampered by the spurious reflections generated by the grid boundaries [9]. These reflections may interfere with the wave packet in the interior region and give unphysical results. Complex absorbing potentials (CAPs) are a widely used way to employ absorbing boundaries in order to eliminate such reflections: the wave function is damped by an imaginary potential in the region near the end of the grid $[10,11]$.

While in the many-body Schrödinger equation, such a CAP is simply added to the Hamiltonian, it is less obvious how the CAP should be implemented on the level of the mean-field approximation. In the present work, we investigate two possibilities and we show that they lead to different results: (i) the CAP is added to the full Hamiltonian and the equations of motion are derived using the variational principle for the resulting Hamiltonian; (ii) the CAP is added directly to the effective potentials in the single-particle equations. Since the KS equations of TDDFT are not necessarily derived from a variational principle, possibility (ii) is the natural way to include absorbing boundaries in TDDFT. For this reason, approach (ii) will be termed the TDDFT approach in the following, while possibility (i) is termed the variational approach.

Atomic units are used in this article.

\section{THEORY}

To demonstrate the difference between the two methods of implementing absorbing boundaries, we apply them explicitly to two example systems. The first one is a one-dimensional $\mathrm{H}_{2}{ }^{+}$molecular ion with coupled electronic and nuclear motion [12]. In the variational approach with absorbing boundaries, the model system is characterized by the Hamiltonian,

$$
\begin{gathered}
\widehat{H}=\widehat{H}_{\mathrm{n}}+\widehat{H}_{\mathrm{e}}+W_{\mathrm{en}}(x, R), \\
\widehat{H}_{\mathrm{e}}=\widehat{T}_{\mathrm{e}}+V_{\text {cap }}^{\mathrm{e}}(x)+q_{\mathrm{e}} x E(t), \\
\widehat{H}_{\mathrm{n}}=\widehat{T}_{\mathrm{n}}+V_{\text {cap }}^{\mathrm{n}}(R)+W_{\mathrm{nn}}(R),
\end{gathered}
$$

with $\widehat{T}_{\mathrm{n}}=-\partial_{R}^{2} /\left(2 \mu_{\mathrm{n}}\right)$ and $\widehat{T}_{\mathrm{e}}=-\partial_{x}^{2} /\left(2 \mu_{\mathrm{e}}\right)$ being the nuclear and the electronic kinetic-energy operator, respectively. Here, $x$ and $R$ denote the electron coordinate and the 
internuclear distance, $\mu_{\mathrm{n}}$ and $\mu_{\mathrm{e}}$ are the reduced masses, $q_{\mathrm{e}}=\left(2 m_{\mathrm{n}}+2\right) /\left(2 m_{\mathrm{n}}+1\right)$ is the reduced charge, and $E(t)$ is the electric field of the laser pulse. For the interactions $W_{\mathrm{en}}$ and $W_{\text {nn }}$, we use soft-core potentials as specified in [12]. The CAPs in the electron and the nuclear direction are $V_{\text {cap }}^{\mathrm{e}}$ and $V_{\text {cap }}^{\mathrm{n}}$, respectively. These CAPs are located near the boundaries of the numerical grid. Apart from annihilating the outgoing wave packets, CAPs may also produce unwanted reflections [13,14] and hence one must ensure that the absorbing boundaries do not change the relevant physics. In the present calculation, we use the shape $V_{\text {cap }}(x)=-i \eta\left|x-x_{\mathrm{c}}\right|^{b} \Theta\left( \pm\left(x-x_{\mathrm{c}}\right)\right)$. The parameters $x_{\mathrm{c}}, \eta$, and $b$ denote the starting point, strength, and order of the CAP, respectively. $\Theta$ denotes the Heaviside step function. When the positive sign is used, the CAP lies to the right of $x_{\mathrm{c}}$, otherwise it is located to the left of $x_{\mathrm{c}}$. The laser field is given by $E(t)=E_{0} f(t) \sin (\omega t)$, where $\omega$ is the laser frequency and $E_{0}$ is the peak amplitude. We use a laser wavelength of $800 \mathrm{~nm}$ and an intensity of $3 \times 10^{14} \mathrm{~W} / \mathrm{cm}^{2}$. The envelope function $f(t)$ has a 2-3-2 shape with a two-cycle $\sin ^{2}$-shaped switch-on, followed by a three-cycle plateau, and a two-cycle $\sin ^{2}$-shaped switch-off. Field-free propagation is added after the pulse so that the total propagation time is $35 \mathrm{fs}$. The wave function $\Psi(x, R, t)$ is represented on four different grid sizes, namely $N_{R} \times N_{x}=$ $256 \times 512,256 \times 1024,256 \times 2048$, and $256 \times 4096$ points, respectively. The spatial step sizes for the internuclear distance and for the electron coordinate are $d R=0.1$ a.u. and $d x=$ 0.2 a.u., respectively. The parameters $x_{\mathrm{c}}=20$ a.u., $\eta=0.35$, and $b=3$ are used for the CAP in the nuclear direction. The parameters $\eta=0.30$ and $b=3$ are used for the CAP in the electronic direction, and the parameter $x_{\mathrm{c}}$ for the four different grid sizes is \pm 45 a.u., \pm 90 a.u., \pm 190 a.u., and \pm 390 a.u., respectively. The ground state is obtained by imaginary-time propagation [15].

In the TDH approximation via the variational approach for $\mathrm{H}_{2}{ }^{+}$, we make the ansatz,

$$
\Psi(x, R, t)=\chi(R, t) \phi(x, t),
$$

where the constraint $\left\langle\chi \mid \partial_{t} \chi\right\rangle=0$ is applied so that $\chi$ remains normalized throughout the propagation. Using the Dirac-Frenkel principle $\left\langle\delta \Psi\left|H-i \partial_{t}\right| \Psi\right\rangle=0$ [16] we obtain the equations of motion,

$$
\begin{aligned}
i \partial_{t} \phi(x, t)=\left[\widehat{H}_{\mathrm{e}}\right. & \left.+\left\langle\chi\left|W_{\mathrm{en}}\right| \chi\right\rangle+\left\langle\chi\left|\widehat{H}_{\mathrm{n}}\right| \chi\right\rangle\right] \phi(x, t), \\
i \partial_{t} \chi(R, t)= & {\left[\widehat{H}_{\mathrm{n}}+\frac{\left\langle\phi\left|W_{\mathrm{en}}\right| \phi\right\rangle}{\langle\phi \mid \phi\rangle}-\left\langle\chi\left|\widehat{H}_{\mathrm{n}}\right| \chi\right\rangle\right.} \\
& \left.-\frac{\left\langle\chi \phi\left|W_{\mathrm{en}}\right| \chi \phi\right\rangle}{\langle\phi \mid \phi\rangle}\right] \chi(R, t) .
\end{aligned}
$$

A slightly different version of the ansatz in Eq. (4) can be written as

$$
\Psi(x, R, t)=a(t) \chi(R, t) \phi(x, t) .
$$

Here $a(t)$ is a time-dependent complex number that allows one to freely choose the phases of the single-particle orbitals $\chi(R, t)$ and $\phi(x, t)$. We can then put constraints on the singleparticle orbitals so that both remain normalized throughout the propagation and the coefficient $a(t)$ is left free to evolve. Although the resulting equations of motion are slightly different, they yield the same total wave function as Eqs. (5) and (6).

In the Hartree TDDFT approach for $\mathrm{H}_{2}{ }^{+}$, the electronnuclear interaction is approximated by the classical electrostatic potentials caused by the respective charge distributions, and correlation contributions are neglected. Self-interaction terms will be omitted. There is no true exchange as the degrees of freedom under consideration are distinguishable. We add the CAPs directly to the KS equations. This is an ad-hoc procedure since TDDFT is defined rigorously only for real potentials. The resulting equations in this case are

$$
\begin{aligned}
i \partial_{t} \phi(x, t) & =\left[\widehat{H}_{\mathrm{e}}+\left\langle\chi\left|W_{\mathrm{en}}\right| \chi\right\rangle\right] \phi(x, t), \\
i \partial_{t} \chi(R, t) & =\left[\widehat{H}_{\mathrm{n}}+\left\langle\phi\left|W_{\mathrm{en}}\right| \phi\right\rangle\right] \chi(R, t),
\end{aligned}
$$

where the CAPs are included in $\widehat{H}_{\mathrm{e}}$ and $\widehat{H}_{\mathrm{n}}$ as before.

The second system under investigation is a one-dimensional helium atom with two interacting electrons [17]. In the variational approach with absorbing boundaries, the Hamiltonian $\widehat{H}$ is given by

$$
\begin{gathered}
\widehat{H}=\widehat{H}_{1}+\widehat{H}_{2}+W_{\mathrm{ee}}\left(x_{1}, x_{2}\right), \\
\widehat{H}_{j}=\widehat{T}_{j}+V\left(x_{j}\right)+V_{\text {cap }}^{\mathrm{e}}\left(x_{j}\right)+x_{j} E(t), \quad j=1,2,
\end{gathered}
$$

with $\quad W_{\text {ee }}\left(x_{1}, x_{2}\right)=1 / \sqrt{1+\left(x_{1}-x_{2}\right)^{2}}, \quad \widehat{T}_{j}=-\partial_{x_{j}}^{2} / 2$, and $V(x)=-2 / \sqrt{1+x^{2}}$. We use the same laser field and grid parameters as in the case of $\mathrm{H}_{2}{ }^{+}$except that we increase the intensity to $2 \times 10^{15} \mathrm{~W} / \mathrm{cm}^{2}$ and we use the grid sizes 512 and 16384 points for the electron coordinate. The CAP $V_{\text {cap }}^{\mathrm{e}}$ has the same form as previously mentioned, using $x_{\mathrm{c}}= \pm 46$ and $x_{\mathrm{c}}= \pm 1475$, respectively.

The variational approach for the two-electron system makes the ansatz,

$$
\Psi\left(x_{1}, x_{2}, t\right)=\phi\left(x_{1}, t\right) \phi\left(x_{2}, t\right),
$$

and leads via the Dirac-Frenkel principle to the equation of motion,

$i \partial_{t} \phi\left(x_{1}, t\right)=\left[\widehat{H}_{1}+\frac{\left\langle\phi\left|W_{\mathrm{ee}}\right| \phi\right\rangle_{2}}{\langle\phi \mid \phi\rangle}-\frac{\left\langle\phi \phi\left|W_{\mathrm{ee}}\right| \phi \phi\right\rangle}{2\langle\phi \mid \phi\rangle^{2}}\right] \phi\left(x_{1}, t\right)$,

where the subscript 2 indicates that $\phi$ appears with argument $x_{2}$ in the expectation value, which is an integral over $x_{2}$. In the TDDFT approach for this system we have

$$
i \partial_{t} \phi\left(x_{1}, t\right)=\left[\widehat{H}_{1}+\left\langle\phi\left|W_{\mathrm{ee}}\right| \phi\right\rangle_{2}\right] \phi\left(x_{1}, t\right) .
$$

Here, we have approximated the KS potential by the selfinteraction corrected Hartree potential, which is for a twoelectron system identical to exchange-only TDDFT as well as the Hartree-Fock approximation [18].

\section{DISCUSSION OF THE EQUATIONS}

In this section, we compare the equations of motion derived from the variational and the TDDFT approaches. Equations (5) and (8) have similar form. The difference is that the potential in Eq. (5) includes a time-dependent constant $\left\langle\chi\left|\widehat{H}_{\mathrm{n}}\right| \chi\right\rangle$. Although the mean-field term $\left\langle\chi\left|W_{\mathrm{en}}\right| \chi\right\rangle$ is formally the same in both equations, we note that the norm of the orbital $\chi$ may decrease with time in the TDDFT 
approach while in the variational approach, we have enforced $\langle\chi \mid \chi\rangle=1$ by construction. Comparing Eqs. (6) and (9) we find that the mean-field $\left\langle\phi\left|W_{\text {en }}\right| \phi\right\rangle /\langle\phi \mid \phi\rangle$ of Eq. (6) includes a factor $1 /\langle\phi \mid \phi\rangle$ which is absent from Eq. (9). Application of absorbing boundaries causes $\langle\phi \mid \phi\rangle$ to be time dependent. This affects the system dynamics significantly. Effectively, all mean fields in the variational approach are generated by normalized densities. The potential in Eq. (6) includes two time-dependent constants: the contribution $-\left\langle\chi\left|\widehat{H}_{\mathrm{n}}\right| \chi\right\rangle$ will exactly compensate the effect of the constant in Eq. (5) once $\phi$ and $\chi$ are multiplied together; the last potential term serves to avoid double counting of the electron-nuclear interaction in the total wave function. In the case of the two-electron system, we compare Eqs. (13) and (14). As previously mentioned, we note that the variational mean field includes a factor $1 /\langle\phi \mid \phi\rangle$, which will cause differences between the two approaches. The last potential term in Eq. (13) prevents double counting of the electron-electron interaction.

Insight is also gained by observing that a variational solution $\phi(x, t)$ multiplied by a constant is again a solution of the variational equations of motion. Although this is a convenient mathematical property, it means that the magnitude of the orbital norm does not have a physical effect on the time evolution. This is different in the TDDFT approach, where the magnitude of the norm does affect the mean-field interaction.

We emphasize that in the absence of absorbing boundaries the orbitals obtained from the variational and the TDDFT approaches will differ only by phase factors. Therefore it is reasonable to construct a total wave function using Eqs. (4) and (12) also for the TDDFT orbitals. All observables shown below are independent of the global phase of the total wave function.

\section{RESULTS}

In Figs. 1(a) and 1(b) we plot the modulus squared of the autocorrelation function $C(t)=\langle\Psi(0) \mid \Psi(t)\rangle$, defined as the overlap of $\Psi(t)$ with the initial ground state $\Psi(0)$. This describes the probability that the system still remains in the ground state. For $\mathrm{H}_{2}{ }^{+}$we do not see a noticeable difference between the variational and the TDDFT calculations on the small grid. The larger grids give the same result (not shown). This may seem compelling enough to conclude that, irrespective of grid size, both approaches perform equally well. For helium, however, we find a striking difference between the two approaches: on the small grid, the variational approach makes the autocorrelation function almost go to zero while the TDDFT approach, as well as the calculations on the large grid, lead to a final value above 0.1 . This means that the small-grid variational approach predicts significantly higher ionization yields. The right-hand side of the figure shows the orbital norm squares as a function of time. The norm of $\chi$ stays practically equal to one because the nuclei do not reach the boundary within the time scale of the calculation. The norm of $\phi$ decreases with time in all cases. Below we demonstrate that this decrease causes differences between the two approaches also in the case of $\mathrm{H}_{2}{ }^{+}$when other observables are plotted. Another reason to investigate the case of $\mathrm{H}_{2}{ }^{+}$in more detail is the clearer separation of cause and effect: the loss of electron probability leads to changes in the nuclear dynamics.

Fig. 2 shows the time-dependent expectation value of the internuclear distance $\langle R\rangle=\int_{0}^{R_{\max }} R d R \int_{x_{\min }}^{x_{\max }}|\Psi(x, R, t)|^{2} d x$, where $R_{\max }$ is the end of the grid in nuclear direction. Note that $\langle R\rangle$ is calculated without normalization of $\Psi$. In Fig. 2(a), we see that the variational and the TDDFT calculations closely follow each other until the end of the pulse, but at later times they begin to deviate. This deviation can be understood as follows. At the beginning of the propagation both orbitals have unit norm. As the system is ionized, the norm of the orbital $\phi(x, t)$ begins to decrease; see Fig. 1(b). This in turn will give a more tightly bound $\langle R\rangle$ in the variational approach compared to the TDDFT approach because the mean fields are effectively increased by factor $1 /\langle\phi \mid \phi\rangle$ [see Eq. (6)]. Comparing the TDDFT calculations in Figs. 2(a)-2(d), we find that $\langle R\rangle$ is almost independent of the grid size. The variational calculation only gradually improves and converges to the TDDFT calculation with increasing grid size.
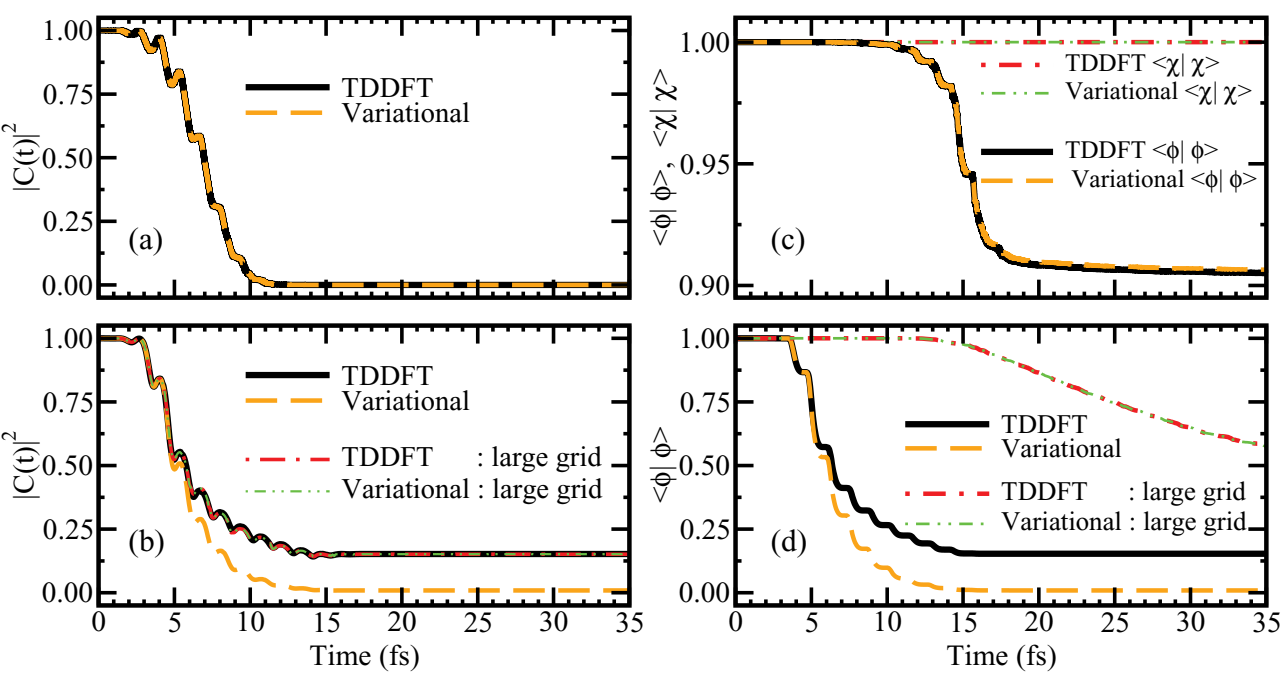

FIG. 1. (Color online) (Left) Modulus squared $|C(t)|^{2}$ of the autocorrelation function for (a) $\mathrm{H}_{2}{ }^{+}$and (b) helium. (Right) Orbital norm squares for (c) $\mathrm{H}_{2}{ }^{+}$and (d) helium. The electronic grid size is 512 points except for the helium large grid with 16384 points. 

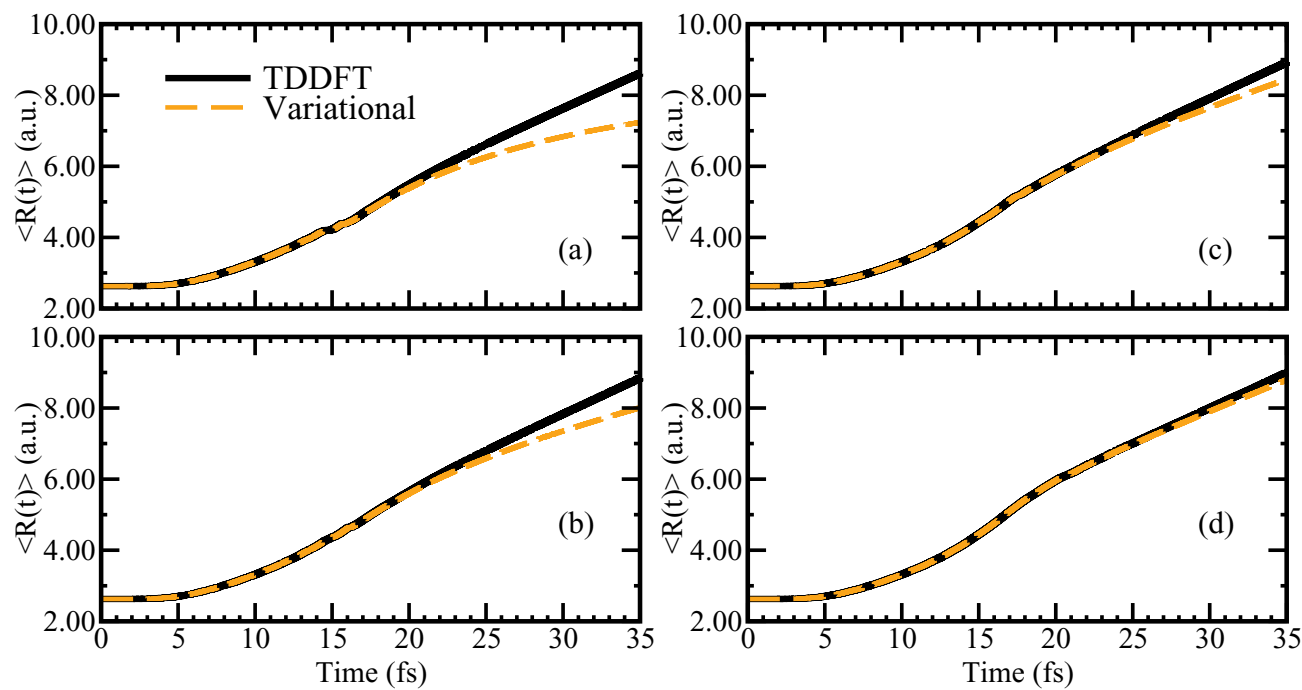

FIG. 2. (Color online) Time evolution of the mean internuclear distance $\langle R\rangle(t)$ in $\mathrm{H}_{2}{ }^{+}$for (a) $256 \times 512$, (b) $256 \times 1024$, (c) $256 \times 2048$, and (d) $256 \times 4096$ grid points.

Figure 3 shows the time evolution of the nuclear density $\rho_{n}(R, t)=\int_{x_{\operatorname{man}}}^{x_{\max }}|\Psi(x, R, t)|^{2} d x$ with $x_{\min }, x_{\max }$ denoting the grid boundaries. They corroborate the conclusions drawn in the previous paragraph. All the TDDFT calculations show that the molecule is stretched to an internuclear distance of more than 9 a.u. at the end of the propagation; see Figs. 3(c) and 3 (d). On the other hand, in the variational calculation for the small grid, the molecule is stretched only to a distance of 8 a.u. in the nuclear direction; see Fig. 3(a). It gradually improves to the level of TDDFT calculation with larger grid sizes as seen in Fig. 3(b).
Fig. 4 shows the time evolution of the electronic density $\rho_{e}(x, t)=\int_{0}^{R_{\max }}|\Psi(x, R, t)|^{2} d R$ for the grid points $256 \times 512$ and $256 \times 4096$, respectively. We do not see striking differences between the two approaches. This can be understood from the fact that $\langle\chi \mid \chi\rangle$ is similar in both approaches [see Fig. 1(b)], leading to similar mean fields in Eqs. (5) and (8). After the end of the pulse, the shape of the density indicates that the system predominantly undergoes dissociation with the electron bound to the nuclei. In Figs. 4(a) and 4(b), we observe that the density at the end of the propagation is located at about 4 and 5 a.u., respectively.
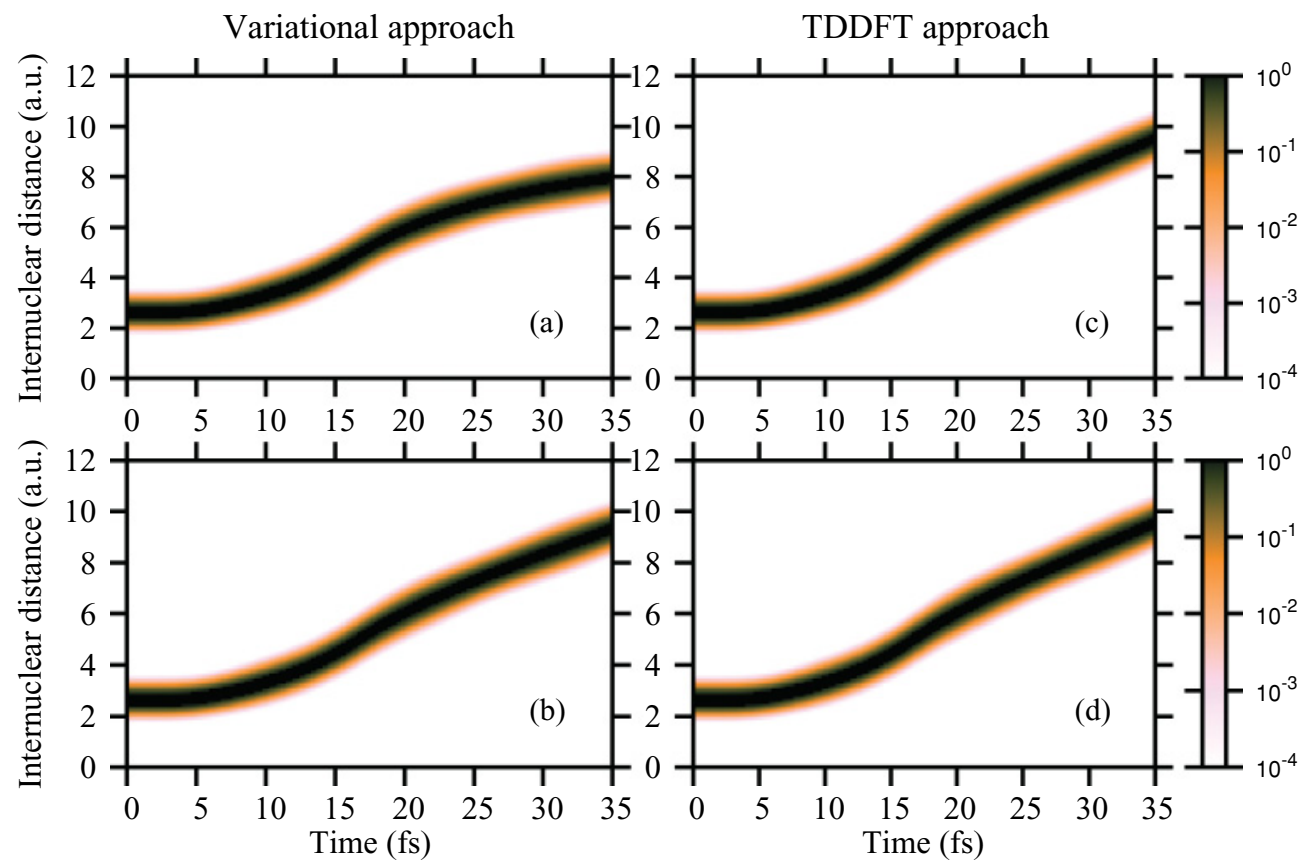

FIG. 3. (Color online) Time evolution of the nuclear density in $\mathrm{H}_{2}{ }^{+}$for (a) $256 \times 512$, (b) $256 \times 4096$, (c) $256 \times 512$, and (d) $256 \times 4096$ grid points. 


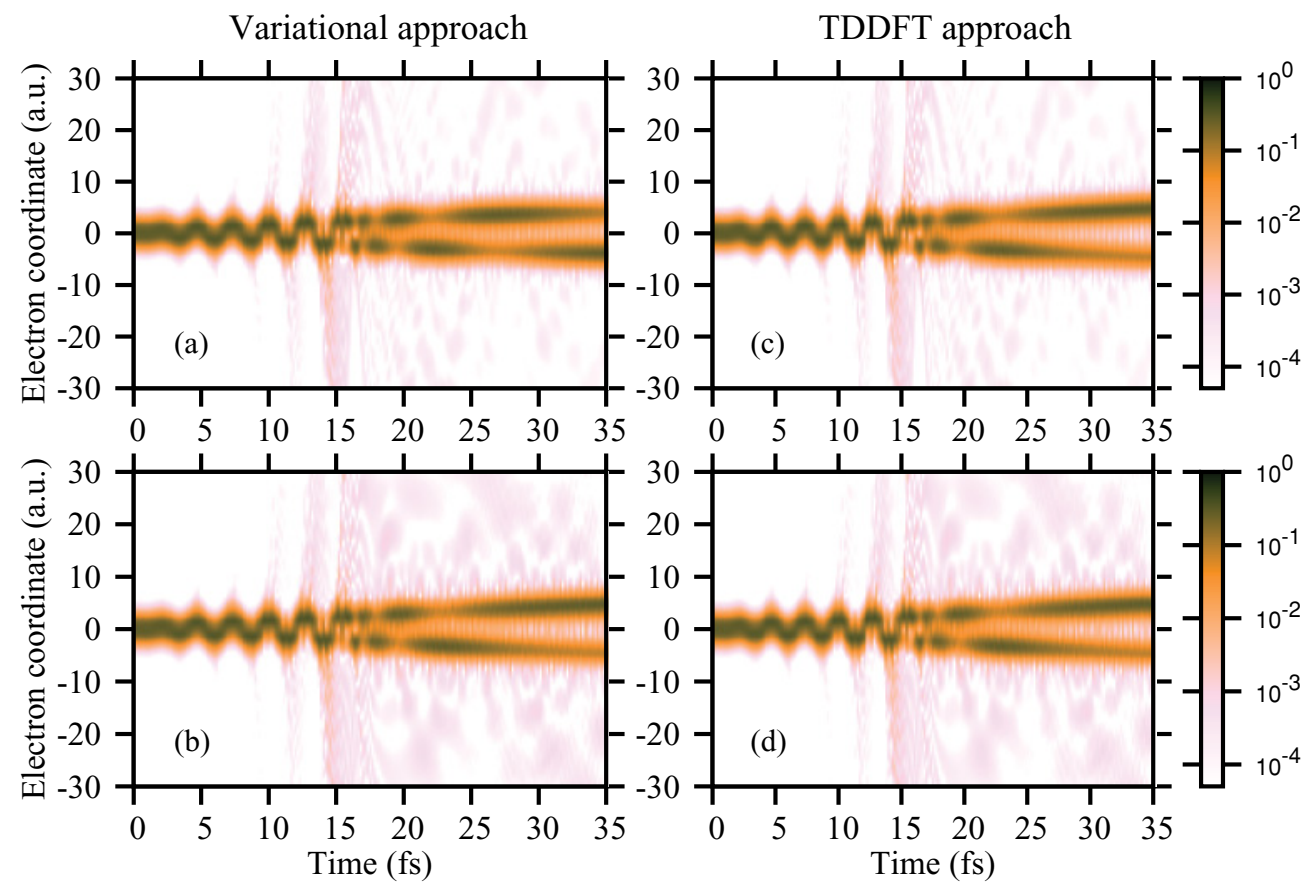

FIG. 4. (Color online) Time evolution of the electronic density in $\mathrm{H}_{2}{ }^{+}$for (a) $256 \times 512$, (b) $256 \times 4096$, (c) $256 \times 512$, and (d) $256 \times 4096$ grid points.

This difference is consistent with the nuclear density plots in Figs. 3(a) and 3(b).

\section{CONCLUSION}

We have presented two different approaches for including absorbing boundaries in the mean-field approximation. Adding the absorbing boundaries directly in the single-particle equations (termed here as the TDDFT approach) yields results almost independent of the grid size while the variational approach leads to deviations for small grids. For example, the variational approach overestimates the ionization probability in a two-electron system. Therefore, although the variational approach seems more systematic and sound at first sight, it is inferior to the ad-hoc TDDFT approach. The reason lies in the unphysical increase of the mean-field terms upon absorption of density at the grid boundary. This behavior arises since the variational equations of motion are derived by using a product ansatz for the total wave function. For example, the total wave function is invariant under multiplying two of the single-particle orbitals by factors $\alpha$ and $1 / \alpha$, respectively. This can be interpreted such that the magnitudes of the individual orbital norms cannot have physical consequences in the variational approach. Our finding is important in view of the widespread use of effective-potential methods. We expect that a similar effect is present in the multiconfiguration time-dependent Hartree [19] and Hartree-Fock [20] methods in the case that only few configurations are included.

\section{ACKNOWLEDGMENT}

We thank the Deutsche Forschungsgemeinschaft for funding the Centre for Quantum Engineering and Space-Time Research (QUEST).
[1] P. Mulser and D. Bauer, High Power Laser-Matter Interaction (Springer, Berlin Heidelberg, 2010)

[2] A. D. McLachlan, Mol. Phys. 8, 39 (1964).

[3] M. A. L. Marques, C. A. Ullrich, F. Nogueria, A. Rubio, K. Burke, and E. K. U. Gross, Time-Dependent Density Functional Theory, Lect. Notes Phys. 706 (Springer, Berlin Heidelberg, 2006).

[4] T. C. Li and P. Q. Tong, Phys. Rev. A 34, 529 (1986).

[5] O. Butriy, H. Ebadi, P. L. de Boeij, R. van Leeuwen, and E. K. U. Gross, Phys. Rev. A 76, 052514 (2007).

[6] W. Kohn and L. J. Sham, Phys. Rev. 140, A1133 (1965).

[7] A. L'Huillier, K. J. Schafer, and K. C. Kulander, J. Phys. B 24, 3315 (1991).
[8] D. B. Milošević, G. G. Paulus, D. Bauer, and W. Becker, J. Phys. B 39, R203 (2006).

[9] J. L. Krause, K. J. Schafer, and K. C. Kulander, Phys. Rev. A 45, 4998 (1992).

[10] R. Kosloff and D. Kosloff, J. Comput. Phys. 63, 363 (1986).

[11] C. Leforestier and R. E. Wyatt, J. Chem. Phys. 78, 2334 (1983).

[12] K. C. Kulander, F. H. Mies, and K. J. Schafer, Phys. Rev. A 53, 2562 (1996).

[13] U. V. Riss and H.-D. Meyer, J. Phys. B 26, 4503 (1993).

[14] S. Scheit, H.-D. Meyer, N. Moiseyev, and L. S. Cederbaum, J. Chem. Phys. 124, 034102 (2006).

[15] R. Kosloff and H. Tal-Ezer, Chem. Phys. Lett. 127, 223 (1986). 
[16] J. Broeckhove, L. Lathouwers, E. Kesteloot, and P. Van Leuven, Chem. Phys. Lett. 149, 547 (1988).

[17] D. G. Lappas et al., J. Phys. B 29, L619 (1996).

[18] K. C. Kulander, Phys. Rev. A 36, 2726 (1987).
[19] M. H. Beck, A. Jäckle, G. A. Worth, and H.-D. Meyer, Phys. Rep. 324, 1 (2000).

[20] J. Zanghellini, M. Kitzler, T. Brabec, and A. Scrinzi, J. Phys. B , 37, 763 (2004). 